\title{
Impacto do uso de infográficos como materiais de aprendizagem e suas correlações com satisfação, estilos de aprendizagem e complexidade visual
}

\author{
Kamila Takayama Lyra, Seiji Isotani (orientador) \\ ICMC - Universidade de São Paulo (USP) \\ São Carlos - SP - Brazil \\ kalyra_03@usp.br, sisotani@icmc.usp.br
}

\begin{abstract}
Resumo. Infográficos são materiais baseados em gráficos, textos e técnicas de design gráfico que visam facilitar a aprendizagem de um conjunto de informações. Apesar deste formato ter se popularizado nos últimos anos, verifica-se que são raras as evidências empíricas demonstrando seu real impacto na aprendizagem. Dessa forma, levanta-se a seguinte questão de pesquisa: será que os infográficos são materiais de aprendizagem mais adequados/efetivos do que os materiais tradicionalmente empregados (i.e., gráficos e textos)? Para responder à essa questão, até então sem resposta, desenvolvemos um arcabouço de ferramentas e um protocolo experimental que permite a realização de pesquisas empíricas sobre o uso de infográficos na aprendizagem. Em seguida, realizamos experimentos controlados com 74 alunos de graduação estudando conceitos sobre meio-ambiente utilizando diferentes tipos de materiais. Os resultados obtidos por meio de análises estatísticas indicam que infográficos são mais benéficos que os materiais tradicionais em termos de eficiência de aprendizagem e que outros fatores como a satisfação tem um papel fundamental no aprendizado do aluno. Dessa forma, este trabalho possui três contribuições principais: (i) a produção de resultados empíricos demonstrando os benefícios dos infográficos para a aprendizagem, até então raramente disponíveis à comunidade; (ii) a criação de um arcabouço de ferramentas e um protocolo experimental que permitem a realização de pesquisas quantitativas na área de informática na educação com forte rigor científico e reprodutibilidade; e (iii) o desenvolvimento de um framework para classificação da complexidade, específico para infográficos, até o presente momento inédito na literatura.
\end{abstract}

\begin{abstract}
Infographics are visual materials based on graphic, texts and graphical design techniques which aim at facilitating the information acquisition. Although this material's recent popularity, there are few empirical evidences of its real influence on learning. Therefore, we formulate the following research question: Are infographics more suitableleffective than traditionally used learning materials (i.e., graphics and texts)? In order to answer this ignored question, we developed a framework of tools and an experimental protocol to aid empirical researches on learning by infographics. Next, we run controlled experiments with 74 undergraduate students who studied environment-related subjects by different learning materials formats. As result, the statistical analysis indicated that infographics are better than
\end{abstract}


VI Congresso Brasileiro de Informática na Educação (CBIE 2017)

Anais dos Workshops do VI Congresso Brasileiro de Informática na Educação (WCBIE 2017)

traditionally used learning materials concerning learning efficiency. In addition, other factors, such as satisfaction, had a crucial role on students learning. Thus, this work has three main contributions: (i) empirical results showing the learning benefits of infographics, hardly available to the community until then; (ii) a framework of tools and an experimental protocol to aid the scientific rigor and reproducibility of quantitative research on informatics in education; and (iii) guidelines to classify the complexity of infographics, unprecedented on literature until then.

\section{Introdução}

As tecnologias computacionais têm sido amplamente investigadas quanto a sua capacidade de facilitar o processo de ensino-aprendizagem (Isotani et al., 2009). Apesar disso, professores e desenvolvedores que fazem uso da tecnologia ainda precisam avaliar qual formato dos materiais de aprendizagem a ser utilizado para garantir um melhor aprendizado. Dentre eles, um formato que vem recentemente ganhando popularidade no contexto educacional é o infográfico (contração do termo em inglês information graphic). Infográficos unem elementos de design a dados para ajudar a transmitir informações de modo objetivo (Smiciklas, 2012). A Figura 1 ilustra um exemplo típico de visualização de informação no formato infográfico.

Devido a sua natureza informativa, os infográficos passaram a ser empregados como material de apoio ao ensino. Alguns trabalhos indicam potenciais ganhos na diminuição da carga cognitiva (Bellato, 2013; Diakopoulos et al., 2011), outros quanto a retenção de conhecimento a longo prazo (Toth, 2013; Zhang-Kennedy et al., 2013). Basicamente, os resultados sugerem que o uso de infográficos pode facilitar o aprendizado. No entanto, são raros os trabalhos que fazem estudos empíricos sobre o real impacto deste tipo de visualização no contexto educacional e mais raro ainda são estudos que comparam os infográficos com os materiais tradicionais gráfico+texto e texto puro.

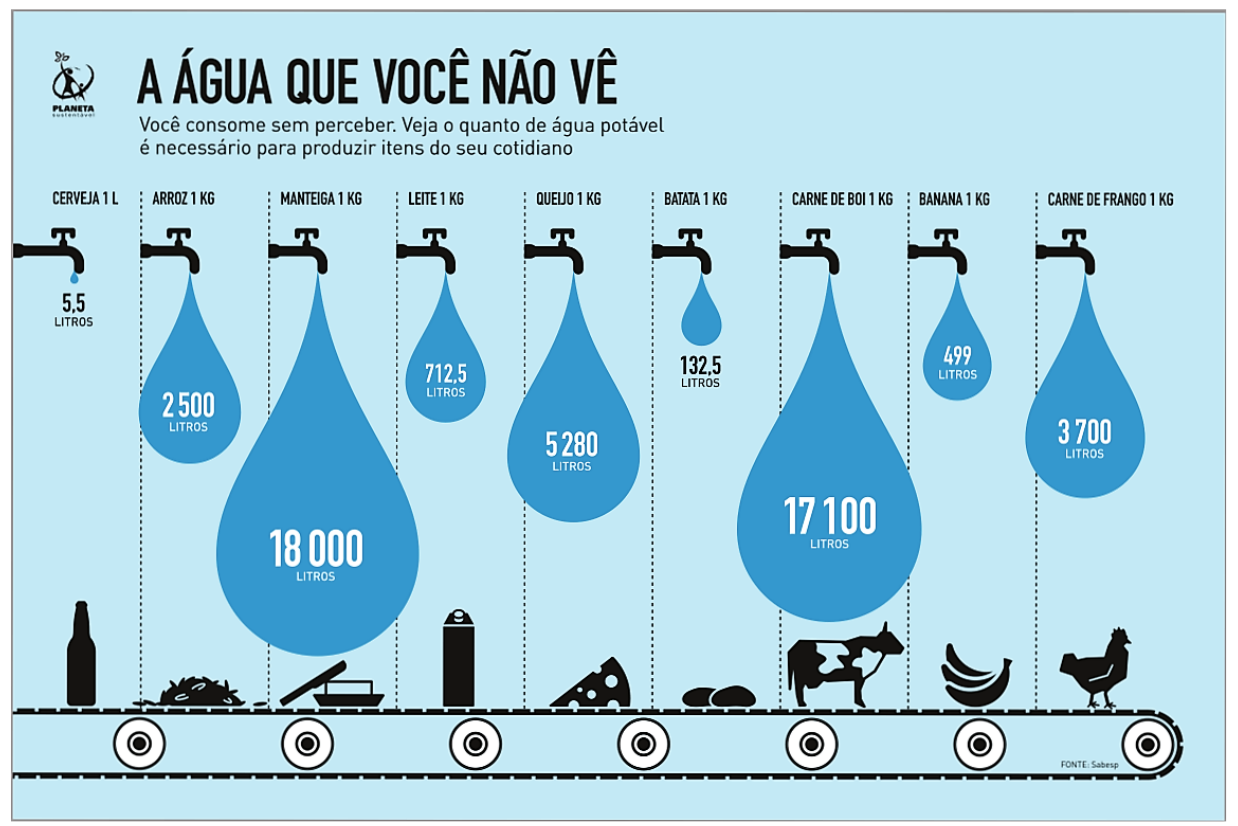

Figura 1. Exemplo de infográfico. Fonte: Planeta Sustentável (2011). 
Ao considerar o apoio cognitivo oferecido pelos infográficos, também é necessário considerar as diferenças na percepção humana ao relacionar-se com esse tipo de visualização. Segundo Gomes (2012), as iniciativas existentes que investigam a influência dos fatores humanos na visualização de dados durante a interação homem-computador ainda estão em fase de aperfeiçoamento, logo, existe um grande potencial de pesquisa nesta temática. Observando a carência de estudos que investiguem a influência de fatores individuais no aprendizado por infográficos, este trabalho também analisa o impacto dos fatores satisfação e estilos de aprendizagem.

Além do tipo de visualização (infográfico, gráfico+texto ou texto puro), existem outros fatores que podem influenciar no aprendizado final. Um fator que também está ligado ao material de aprendizagem é a complexidade da informação presente na visualização. Assim, a complexidade do infográfico é outra variável a ser investigada neste trabalho em relação à interação com o aprendizado.

No intuito de levantar indícios para preencher essas lacunas de pesquisa foram realizados experimentos controlados para comparar o aprendizado por infográficos com o aprendizado por materiais de aprendizagem tradicionais, gráfico+texto e texto puro. Devido a limitação de espaço, neste trabalho será apresentado apenas o experimento final. Além disso, foram feitas assertivas a respeito da influência das variáveis satisfação, estilo de aprendizagem e complexidade do infográfico.

\section{Trabalhos Relacionados}

Dentre os trabalhos experimentais que descrevem o impacto dos infográficos quando utilizados como materiais de aprendizagem, Diakopoulos et al. (2011) e Comello et al. (2016) exploraram o fator gamificação. Apesar de Diakopoulos et al. (2011) observarem as variáveis aprendizado e satisfação, os resultados não comparam infográficos com os formatos tradicionais e sim os diferentes níveis de elementos de jogos nos infográficos. Esse ponto é coberto por Comello et al. (2016) que comparam infográficos gamificados com o formato texto puro e conclui que ao utilizar os infográficos gamificados os sujeitos tiveram melhores resultados de engajamento e compreensão do assunto.

Fora do campo da gamificação, os experimentos descritos por Zhang-Kennedy et al. (2013) e Crick e Hartling (2015) comparam o formato infográfico com o formato tradicional texto puro. Zhang-Kennedy et al. (2013) relatam uma retenção de conhecimento significativamente maior, além de maior sentimento de utilidade e de persuasão de alunos que utilizaram infográficos ao invés de texto. Adicionalmente, Crick e Hartling (2015) destacaram a importância de adequar o formato da visualização ao público alvo. No experimento realizado, foi observada a preferência maior dos usuários com maior formação pelo formato infográficos, enquanto que os usuários com menor formação relataram preferência pelo formato texto. O trabalho de Lee e Kim (2015) foi o único encontrado que compara os infográficos ao formato gráfico+texto. Foi verificado que usuários com menor conhecimento prévio avaliaram mais positivamente as notícias apresentadas na forma de infográficos, ressaltando o poder do apelo visual do infográfico. Apesar de Lee e Kim (2015) compararem os três formatos de materiais de aprendizagem, os alunos não foram avaliados quanto ao aprendizado.

Assim, destaca-se que ainda existem poucos trabalhos que investiguem o real 
impacto dos infográficos no aprendizado. Além disso, nenhum dos trabalhos mencionados verifica a interferência de fatores ligados aos sujeitos, como o estilo de aprendizagem e satisfação, ou de fatores ligados ao infográfico, como a sua complexidade.

\section{Plataforma para Estudo de Infográficos}

Para coletar os dados sobre o aprendizado por infográficos e dar suporte às atividades de experimentação foi desenvolvida uma plataforma online de estudo e avaliação individual. A plataforma e o design experimental escolhido foram validados por meio de um experimento piloto publicado em conferência internacional (Lyra et al., 2016a). A plataforma criada conta com três módulos, um para cada fase do experimento, que serão detalhados a seguir.

Módulo A (Pré-teste): O primeiro módulo permitiu que os alunos se registrassem na plataforma e avaliassem seus conhecimentos iniciais por meio de 45 questões do questionário de conhecimento. Dessa maneira, foi possível comparar a diferença de conhecimento dos sujeitos entre as Fases A e B, ou seja, antes e depois da interação com os materiais de aprendizagem. Em seguida, o módulo A aplicou o questionário N-ILS (Junior, 2012) para identificar os estilos de aprendizagem (visual ou verbal) dos alunos.

Módulo B (Intervenção e Pós-teste): Este módulo possui três versões, cada uma carregando um tipo diferente de material de aprendizagem (infográfico, gráfico+texto ou texto). Os sujeitos foram distribuídos aleatoriamente entre as três versões. O fluxo das atividades executadas nesta fase está ilustrado na Figura 4. O sujeito inicia as atividades realizando sua auto-avaliação de satisfação na ferramenta SAM (Lang, 1980). Em seguida, dá-se início a interação com os materiais de aprendizagem de acordo com a versão do módulo B a que o aluno foi atribuído. Após a visualização de um material, o sujeito responde à uma questão-tema ${ }^{1}$ referente ao material visto. A questão-tema é aplicada entre uma visualização e outra para evitar que os sujeitos passem pelos materiais sem uma leitura adequada. A cada três visualizações e questões-tema, o aluno é questionado novamente sobre sua satisfação com a atividade. Esse fluxo se repete por cinco vezes, até que o aluno visualize os 15 materiais e responda suas 15 questões-tema. A atividade seguinte é o questionário de conhecimento que, nesta fase, tem o objetivo de identificar o conhecimento adquirido logo após a interação com os materiais. Por fim, os sujeitos respondem mais uma vez sua auto avaliação de satisfação na escala SAM, totalizando sete auto-avaliações durante todo o módulo B.

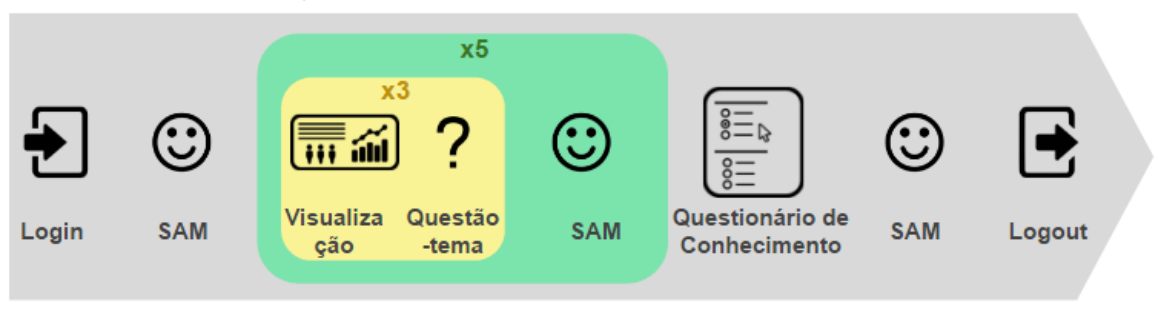

Figura 4. Fluxo de atividades do módulo B.

Módulo C (Teste de Retenção): O último módulo da plataforma aplica o questionário de conhecimento para avaliar o quanto os alunos retiveram de conhecimento sete dias

\footnotetext{
${ }^{1}$ A questão-tema é a mesma para todas as visualizações: "Qual o tema do material apresentado na página anterior?", varia apenas nas alternativas de respostas.
} 
após a interação com os materiais de aprendizagem que ocorreu no módulo B. O código completo da plataforma, os materiais de aprendizagem, os questionários de conhecimento e as ferramentas SAM e N-ILS podem ser encontrados no Lab Package do experimento em https://goo.gl/LT26CL.

\subsection{Framework para classificação da complexidade dos infográficos}

Foi proposto um framework para a classificação da complexidade de infográficos, visto que na literatura não foi encontrado nenhum trabalho com esse propósito. O framework proposto avalia três dimensões de complexidade presentes nos infográficos: a) Complexidade Visual: Baseada no método proposto por Zhu et al. (2007) em que cada atributo (i.e. cor, forma, tamanho, posição, orientação e textura) de cada unidade visual (e.g. um ícone, uma ilustração, uma coluna de um gráfico) recebe uma pontuação de complexidade. A complexidade visual final de um infográfico é dada pela soma da complexidade de todas as suas unidades visuais. b) Complexidade Textual: Foi utilizada a versão para a língua portuguesa do teste de legibilidade Flesch Reading Ease (FRE)(Flesch, 1948; Martins et al., 1996). FRE é o teste utilizado pelo editor de texto Microsoft Word® para calcular a legibilidade de documentos de texto. A fórmula leva em consideração o número de sílabas, de palavras e de frases em um texto. O resultado é dado em uma escala com valores de 0 a 100, sendo que quanto mais alto o valor mais fácil é a leitura do texto. c) Complexidade Conceitual: Fundamentada na Teoria da Complexidade Relacional (Relational Complexity Theory - RCT)(Halford et al., 1998; Andrews e Halford, 2002) foram propostos valores para as complexidades relacionais. Esse valores foram planejados para cumprir a premissa de que relações com mais argumentos são mais complexas do que várias relações menores, desde que o número de argumentos não seja ultrapassado. Mais informações sobre como o framework foi utilizado podem ser encontradas em Lyra (2017).

\section{Execução do experimento final}

O experimento final teve a coleta de dados realizada em duas execuções que seguiram o design experimental publicado em periódico nacional (Lyra et al., 2016b). A primeira ocorreu em março de 2016 e contou com 58 alunos no primeiro semestre do curso de Engenharia de Computação. A segunda execução ocorreu em maio de 2016 e teve como sujeitos 44 alunos do curso de Bacharelado em Sistemas de Informação. Dentre os 102 alunos participantes, apenas os dados de $\mathbf{7 4}$ sujeitos puderam ser considerados. Os 74 sujeitos têm, em média 18,8 $(\sigma=3)$ anos, sendo 68 do sexo masculino e 6 do sexo feminino. O N-ILS identificou 28 alunos verbais e 46 visuais. Durante a Fase B (Intervenção e Pós-teste), 24 alunos foram atribuídos aleatoriamente à infográficos, 23 alunos utilizaram gráficos+texto e 27 utilizaram o formato de material de aprendizagem texto puro. Os dados tabulados podem ser encontrados em https://goo.gl/hWhhvG.

\section{Analisando o impacto dos Infográficos}

Para realizar as análise, foi utilizado o software estatístico SPSS. O teste ANOVA foi utilizado para comparar as médias das medidas de aprendizado, tempo e satisfação entre os três grupos experimentais e verificar se existe diferença estatisticamente significativa $(\mathrm{p}<0,05)$ entre elas. Os resultados e a interpretação dos testes para cada variável investigada são apresentados a seguir. As análises estatísticas completas podem ser encontradas em https://goo.gl/e2ZpTv. 
VI Congresso Brasileiro de Informática na Educação (CBIE 2017)

Anais dos Workshops do VI Congresso Brasileiro de Informática na Educação (WCBIE 2017)

\subsection{O aprendizado}

Foram analisadas as medidas de aprendizado: $\Delta 1$ que representa o quanto um aluno aprendeu da Fase A para a Fase B (B-A), $\Delta 2$ que representa o quanto um aluno perdeu da Fase B para a Fase $\mathrm{C}$ (B-C) e $\Delta 3$ que representa o quanto de conteúdo foi retido da Fase A até a Fase C (C-A).

Pela Tabela 1, é possível ver que o grupo que utilizou gráficos+texto teve as maiores médias para o aprendizado imediato $(\Delta 1)$ e retenção $(\Delta 3)$. No entanto, considerando a medida $\Delta 2$, que representa a taxa de perda de conhecimento da Fase B para a Fase $\mathrm{C}$, o melhor resultado foi obtido pelo grupo que utilizou infográficos. Enquanto que o grupo que mais perdeu conhecimento foi o grupo que utilizou texto. Este pode ser considerado um indício de que infográficos são mais adequados quando o objetivo é aprendizado efetivo, já que a taxa de perda foi a menor entre os três grupos.

Aplicado o teste ANOVA, não foram encontradas diferenças estatísticas significativas entre as médias dos três grupos para nenhuma das medidas $(\Delta 1: \mathrm{F}(2,71)=$ 1,194; $\mathrm{p}=, 309 ; \Delta 2: \mathrm{F}(2,71)=1,125 ; \mathrm{p}=, 330 ; \Delta 3: \mathrm{F}(2,71)=, 877 ; \mathrm{p}=, 420)$. Ou seja, os infográficos foram utilizados como materiais de aprendizagem sem prejuízo ao aprendizado do aluno, obtendo médias de aprendizado semelhantes às médias dos grupos que utilizaram os materiais tradicionais, gráfico+texto e texto puro.

Tabela 1. Medidas de aprendizado por grupo.

\begin{tabular}{|c|c|c|c|c|c|c|}
\hline & \multicolumn{6}{|c|}{ Material de aprendizagem } \\
\hline & \multicolumn{2}{|c|}{ Infográfico } & \multicolumn{2}{|c|}{ Gráfico+Texto } & \multicolumn{2}{|c|}{ Texto puro } \\
\hline & Média & DP & Média & DP & Média & DP \\
\hline$\Delta \mathbf{1}(\mathbf{B}-\mathbf{A})$ & 15,417 & 5,064 & 17,957 & 6,477 & 17,630 & 6,851 \\
\hline$\Delta 2$ (B-C) & 2,000 & 2,064 & 2,130 & 3,167 & 3,000 & 2,496 \\
\hline$\Delta \mathbf{3}(\mathbf{C}-\mathbf{A})$ & 13,417 & 5,429 & 15,826 & 7,328 & 14,630 & 5,884 \\
\hline
\end{tabular}

Apesar de não obter as maiores médias de aprendizado, o grupo de alunos que utilizou infográficos foi o que apresentou o aprendizado mais eficiente, ou seja, aprendeu mais em menos tempo de estudo. A Tabela 2 mostra as medidas de eficiência por grupo, que são resultado da divisão da medida de aprendizado ( $\Delta 1$ e $\Delta 3)$ pelo tempo de estudo do material. Quanto maior o valor, mais eficiente foi a performance do grupo. Essa eficiência confirma o que é argumentado pela literatura sobre o objetivo dos infográficos, que é transmitir conhecimento duradouro de forma simples de ser lida, transformando conhecimento complexo em conhecimento intuitivo (Smiciklas, 2012).

Tabela 2. Medidas de eficiência por grupo.

\begin{tabular}{|l|l|l|l|}
\hline & \multicolumn{3}{|c|}{ Material de aprendizagem } \\
\hline & \multicolumn{1}{|c|}{ Infográfico } & \multicolumn{1}{|c|}{ Gráfico+Texto } & \multicolumn{1}{c|}{ Texto puro } \\
\hline$\Delta \mathbf{1}$ (B-A) / Tempo (s) & $\mathbf{0 , 1 6 6}$ & 0,159 & 0,164 \\
\hline$\Delta \mathbf{3}$ (C-A) / Tempo (s) & $\mathbf{0 , 1 4 5}$ & 0,140 & 0,136 \\
\hline
\end{tabular}

\subsection{A complexidade do infográfico}

As medidas de aprendizado $(\Delta 1$ e $\Delta 3)$ e de tempo, apresentadas na Tabela 3 , são 
coerentes com a classificação de complexidade baixa e média dos infográficos. Por meio do teste ANOVA associado ao teste de Múltiplas Comparações, foi possível observar que as médias de aprendizado imediato $(\Delta 1)$, retenção $(\Delta 3)$ e tempo foram estatisticamente mais altas $(\mathrm{p}=, 000)$ para os infográficos classificados como baixa complexidade do que para os de média complexidade. Porém, a diferença entre as médias referentes às complexidades médias e altas não foram estatisticamente significativas para o aprendizado e retenção $(\Delta 1: \mathrm{p}=, 077 ; \Delta 3: \mathrm{p}=, 187)$. Em outras palavras, os alunos utilizaram menos tempo e aprenderam significativamente mais a partir de infográficos de baixa complexidade do que a partir de infográficos de média e alta complexidade. A partir desses resultados, é possível afirmar que o framework para classificação da complexidade dos infográficos foi preciso quanto à classificação da complexidade baixa, mas necessita ser calibrado quanto à classificação das complexidades média e alta. Outra interpretação possível está ligada a capacidade do formato infográfico de resumir e simplificar informações complexas. Ou seja, a partir de um determinado nível de complexidade, a natureza explicativa do infográfico permite que o aprendizado do aluno seja estável, mesmo que essa complexidade aumente.

Tabela 3. Medidas de aprendizado e tempo por complexidade do infográfico.

\begin{tabular}{|c|c|c|c|c|c|c|}
\hline & \multicolumn{6}{|c|}{ Complexidade dos Infográficos } \\
\hline & \multicolumn{2}{|c|}{ Baixa } & \multicolumn{2}{|c|}{ Média } & \multicolumn{2}{|c|}{ Alta } \\
\hline & Média & DP & Média & DP & Média & DP \\
\hline$\Delta \mathbf{1}(\mathbf{B}-\mathbf{A})$ & 7,417 & 1,954 & 3,208 & 2,187 & 4,792 & 3,148 \\
\hline$\Delta \mathbf{3}(\mathbf{C}-\mathbf{A})$ & 6,500 & 2,246 & 2,833 & 2,200 & 4,083 & 2,842 \\
\hline Tempo (s) & 49,539 & 26,131 & 88,099 & 58,640 & 140,539 & 91,051 \\
\hline
\end{tabular}

\subsection{A satisfação}

Para calcular a média de satisfação de cada grupo (infográfico, gráfico+texto e texto puro) foi considerado um único valor de satisfação de cada sujeito. Esse valor é a média dos valores dados pelo sujeitos nos sete momentos de questionamento da ferramenta SAM. O grupo com a maior média de satisfação foi o grupo gráfico+texto $\left(\mu_{\text {gráfico+texto }}=\right.$ $5,926 ; \sigma=1,610)$ e com o menor valor é o grupo que utilizou texto puro ( $\mu_{\text {texto }}=4,794$; $\left.\sigma=1,923 ; \mu_{\text {infográfico }}=5,422 ; \sigma=1,917\right)$. No entanto, o teste ANOVA não classificou essa diferença entre as médias de satisfação dos grupos como significativas $(\mathrm{F}(2,71)=2,404$; $\mathrm{p}=$,098), ou seja, o material de aprendizagem utilizado pelo aluno não afetou a declaração de prazer.

Apenas para o grupo de alunos que utilizou infográficos foi identificada uma correlação positiva e significativa entre a variável satisfação e a variável tempo de estudo ( $r=, 494 ; p=, 014)$, isto é, o aluno que passou mais tempo visualizando os infográficos também declarou satisfação mais positiva durante a visualização. No entanto, não é possível identificar uma relação causal entre as duas variáveis, ou seja, não é possível afirmar que o aluno passou mais tempo visualizando os infográficos por estar mais satisfeito, nem que o aluno estava mais satisfeito por ter passado mais tempo visualizando os infográficos.

Também foram encontradas correlações positivas e significativas da variável satisfação com a variável número de acertos na Fase C para os grupos texto ( $r=, 399$; 
VI Congresso Brasileiro de Informática na Educação (CBIE 2017)

Anais dos Workshops do VI Congresso Brasileiro de Informática na Educação (WCBIE 2017)

$\mathrm{p}=, 039)$, texto-visuais $(\mathrm{r}=, 660 ; \mathrm{p}=, 004)$ e infográficos-verbais $(\mathrm{r}=, 839 ; \mathrm{p}=, 037)$. É possível afirmar que, para esses grupos, alunos que se declararam mais satisfeitos durante a interação com os materiais de aprendizagem tiveram melhores resultados quanto a retenção de conhecimento.

\subsection{Os Estilos de Aprendizagem}

A Tabela 4 mostra as médias de aprendizado e satisfação dos três grupos experimentais, separando entre alunos visuais e verbais. Contrariando a Teoria dos Estilos de Aprendizagem (Felder e Silverman, 1988), o grupo de alunos que utilizou infográficos foi o que obteve as menores médias de aprendizado $(\Delta 1)$ e retenção $(\Delta 3)$ dentre os visuais. No mesmo sentido, os alunos classificados como verbais não foram beneficiados pelo uso do material puramente textual, pois as melhores médias de alunos verbais são de alunos que utilizaram gráfico+texto. Apesar de não obter os melhores resultados considerando as medidas de aprendizagem $\Delta 1$ e $\Delta 3$, o grupo que utilizou infográficos teve as menores médias de perda de conhecimento $(\Delta 2)$, tanto para o estilo visual quanto para o verbal. A maior média de satisfação entre os alunos visuais é a do grupo que utilizou o formato gráfico+texto. Entre os alunos verbais, o grupo que utilizou infográficos apresentou a maior média de satisfação.

O teste ANOVA não identificou diferenças estatísticas significativas entre as médias das medidas de aprendizado dos três grupos, seja para a amostra de alunos visuais $(\Delta 1: \mathrm{F}(2,43)=1,413 ; \mathrm{p}=, 225 ; \Delta 2: \mathrm{F}(2,43)=, 524 ; \mathrm{p}=, 596 ; \Delta 3: \mathrm{F}(2,43)=$ $1,027 ; \mathrm{p}=, 367)$ ou verbais $(\Delta 1: \mathrm{F}(2,25)=, 076 ; \mathrm{p}=, 927 ; \Delta 2: \mathrm{F}(2,25)=, 670 ; \mathrm{p}=, 520$; $\Delta 3: \mathrm{F}(2,25)=, 148 ; \mathrm{p}=, 863)$. Da mesma forma, a diferença entre as médias de satisfação entre os grupos não foi significativa para alunos visuais $(\mathrm{F}(2,43)=2,392 ; \mathrm{p}=$ ,104), nem para alunos verbais $(\mathrm{F}(2,25)=, 452 ; \mathrm{p}=, 641)$.

Estes resultados oferecem mais subsídios à vertente contrária à Teoria de Estilos de Aprendizagem, que discute a falta de evidências sobre o real impacto dos Estilos de Aprendizagem (Kirschner, 2016). Mesmo quando o formato do material de aprendizagem é correspondente ao estilo, não é possível afirmar que alunos identificados como visuais aprendem mais, ou se sentem mais satisfeitos, apenas por utilizarem um material com mais informações visuais como o infográfico.

Tabela 4. Aprendizado e satisfação por grupo e por estilo de aprendizagem.

\begin{tabular}{|c|c|c|c|c|c|c|c|}
\hline & & \multicolumn{6}{|c|}{ Material de aprendizagem } \\
\hline & & \multicolumn{2}{|c|}{ Infográfico } & \multicolumn{2}{|c|}{ Gráfico+Texto } & \multicolumn{2}{|c|}{ Texto puro } \\
\hline & & Média & DP & Média & DP & Média & DP \\
\hline \multirow[t]{2}{*}{$\Delta \mathbf{1}(\mathrm{B}-\mathrm{A})$} & Visual & 15,167 & 5,079 & 18,545 & 5,820 & 18,118 & 7,347 \\
\hline & Verbal & 16,167 & 5,419 & 17,417 & 7,242 & 16,800 & 6,197 \\
\hline \multirow[t]{2}{*}{$\Delta \mathbf{2}(\mathrm{B}-\mathrm{C})$} & Visual & 2,222 & 2,074 & 2,455 & 3,078 & 3,118 & 2,891 \\
\hline & Verbal & 1,333 & 2,066 & 1,833 & 3,353 & 2,800 & 1,751 \\
\hline \multirow[t]{2}{*}{$\Delta \mathbf{3}(\mathbf{C}-\mathbf{A})$} & Visual & 12,944 & 4,893 & 16,091 & 7,286 & 15,000 & 6,344 \\
\hline & Verbal & 14,833 & 7,139 & 15,583 & 7,681 & 14,000 & 5,270 \\
\hline \multirow[t]{2}{*}{ Satisfação } & Visual & 5,238 & 1,561 & 6,091 & 1,661 & 4,614 & 1,971 \\
\hline & Verbal & 5,976 & 2,852 & 5,775 & 1,619 & 5,101 & 1,899 \\
\hline
\end{tabular}




\section{Conclusão}

Com o objetivo de comparar o aprendizado por meio de infográficos com o aprendizado por gráfico+texto e texto puro, foi planejado e executado um experimento controlado com 74 alunos de graduação. Os alunos foram avaliados quanto à aprendizagem imediata, retenção e perda de conhecimento, satisfação e estilos de aprendizagem. Como contribuição à comunidade científica, este trabalho de mestrado deixa assertivas sobre o impacto e uso adequado do formato de material de aprendizagem conhecido como infográfico. Foram produzidas evidências baseadas em dados de que infográficos podem ser tão bons quanto os materiais tradicionais. Além disso, foram encontrados indícios de que o aprendizado por infográficos pode ser mais eficiente, visto que este foi o grupo em que os alunos utilizaram menos tempo para estudar os materiais.

Assim como foi discutido por Kirschner (2016), não foram encontradas evidências empíricas de que os estilos de aprendizagem visual ou verbal têm impacto na aprendizagem ou na satisfação dos alunos com o formato do material utilizado. Em relação a satisfação declarada pelo aluno, foram encontradas correlações significativas entre a satisfação e a retenção de conhecimento, indicando que satisfações positivas resultam em retenção de conhecimento maior. Em suma, os resultados mostraram a satisfação do aluno como o fator mais influente no aprendizado, acima dos estilos de aprendizagem ou do formato do material.

Uma outra contribuição deste trabalho foi of framework para analisar e classificar a complexidade dos infográficos. Até o presente momento não se tem conhecimento na literatura de nenhum outro framework para classificação da complexidade dos infográficos. Além de classificar, as dimensões avaliadas pelo framework podem servir como parâmetro para que desenvolvedores de infográficos aumentem ou diminuam a complexidade de seus materiais. A classificação de complexidade, juntamente com o fato de o aprendizado não ser significativamente menor para infográficos de complexidade alta, devem ser considerados na tomada de decisão sobre quando é viável ou não gerar uma visualização para transmitir um conteúdo.

Este trabalho deixa como contribuição também um arcabouço de ferramentas juntamente com o protocolo experimental utilizado. Este conjunto de materiais permite que a comunidade replique os procedimentos em busca de maiores evidências sobre o uso de infográficos na aprendizagem, ou adapte e aplique os materiais no contexto de outras pesquisas. Ainda, os dados e as análises obtidas no experimento são de livre acesso, possibilitando validação dos resultados e futuras análises. Todos os materiais podem ser encontrados no Lab Package do experimento em https://goo.gl/LT26CL.

Espera-se que as contribuições aqui apresentadas possam ser aplicadas tanto de modo informal, por professores e alunos no dia a dia do aprendizado, como de modo formal, por desenvolvedores de materiais e de ambientes virtuais de aprendizagem, com o objetivo de tornar o processo de aprendizagem mais efetivo. 
VI Congresso Brasileiro de Informática na Educação (CBIE 2017)

Anais dos Workshops do VI Congresso Brasileiro de Informática na Educação (WCBIE 2017)

\section{Agradecimentos}

Kamila Takayama Lyra agradece à CNPq e à CAPES pelo apoio financeiro na condução dessa pesquisa.

\section{Referências}

Andrews, G.; Halford, G. S. (2002) A cognitive complexity metric applied to cognitive development.Cognitive Psychology, v. 45, n. 2, p. 153-219.

Bellato, N. (2013) Infographics: A visual link to learning. ACM eLearn Magazine. Article No 1. Disponível em: http://dx.doi.org/10.1145/2556598.2556269

Comello, M. L. G.; Qian, X.; Deal, A. M.; Ribisl, K. M.; Linnan, L. A.; Tate, D. F. (2016) Impact of game-inspired infographics on user engagement and information processing in an ehealth program. Journal of Medical Internet Research, v. 18, n. 9.

Crick, K.; Hartling, L. (2015) Preferences of knowledge users for two formats of summarizing results from systematic reviews: Infographics and critical appraisals. PLoS ONE, v. 10, n. 10, p. $1-8$.

Diakopoulos, N.; Kivran-swaine, F.; Naaman, M. (2011) Playable Data: Characterizing the Design Space of Game-y Infographics. ACM Conference on Human Factors in Computing Systems, p. 1717-1726.

Felder, R. M.; Silverman, L. K. (1988) Learning and teaching styles in engineering education. Engineering education, v. 78, n. 7, p. 674-681.

Flesch, R. (1948) A new readability yardstick. Journal of applied psychology, American Psychological Association, v. 32, n. 3, p. 221.

Gomes, L. F. O. (2012) Influência da perceção humana no processo de visualização de dados. Departamento de Engenharia Mecânica - Universidade do Porto.

Halford, G. S.; Wilson, W. H.; Phillips, S. (1998) Processing capacity defined by relational complexity: implications for comparative, developmental, and cognitive psychology. The Behavioral and brain sciences, v. 21, n. 6, p. 803-831.

Isotani, S.; Mizoguchi, R.; Bittencourt, I. I.; Costa, E. (2009) Estado da arte em web semântica e web 2.0: potencialidades e tendências da nova geração de ambientes de ensino na internet. Brazilian Journal of Computers in Education, v. 17, n. 01, p. 30.

Junior, N. V. (2012) Planejamento de um ambiente virtual de aprendizagem baseado em interfaces dinâmicas e uma aplicação ao estudo de potência elétrica. Tese (Doutorado) UNESP, Ilha Solteira, 232p.

Kirschner, P. A. (2016) Stop propagating the learning styles myth. Computers \& Education, Elsevier Ltd, v. 106, p. 166-171.

Lang, P. (1980) Self-assessment manikin. Gainesville, FL: The Center for Research in Psychophysiology, University of Florida.

Lee, E.-j.; Kim, Y. W. (2015) Effects of infographics on news elaboration, acquisition, and evaluation: Prior knowledge and issue involvement as moderators. New Media \& Society, p. 1579-1598.

Lyra, K. T. (2017) Impacto do uso de infográficos como materiais de aprendizagem e suas correlações com satisfação, estilos de aprendizagem e complexidade visual. Dissertação 
VI Congresso Brasileiro de Informática na Educação (CBIE 2017)

Anais dos Workshops do VI Congresso Brasileiro de Informática na Educação (WCBIE 2017)

(Mestrado) — ICMC, USP, São Carlos, 167p. DOI: 10.13140/RG.2.2.31184.61444

Lyra, K. T.; Isotani, S.; Reis, R. C.; Marques, L. B.; Pedro, L. Z.; Jaques, P. A.; Bittencourt, I. I. (2016a) Infographics or graphics+text: Which material is best for robust learning? IEEE International Conference on Advanced Learning Technologies, p. 366-370.

Lyra, K. T.; Oliveira, B. R.; Reis, R. C.; Cruz, W. M.; Nakagawa, E. Y.; Isotani, S. (2016b) Infográficos versus Materiais de Aprendizagem Tradicionais: uma Investigação Empírica. RENOTE, v. 14, n. 2.

Martins, T. B. F.; Ghiraldelo, C. M.; Nunes, M. G. V.; Júnior, O. N. O. (1996) Readability Formulas Applied to Textbooks in Brazilian Portuguese. p. 1-12.

Smiciklas, M. (2012) The Power of Infographics: Using Pictures to Communicate and Connect with Your Audiences. Que Publishing.

Toth, C. (2013) Revisiting a Genre: Teaching Infographics in Business and Professional Communication Courses. Business Communication Quarterly, v. 76, n. 4, p. 446-457.

Zhang-kennedy, L.; Chiasson, S.; Biddle, R. (2013) Password advice shouldn't be boring: Visualizing password guessing attacks. eCrime Researchers Summit, p. 1-11.

Zhu, Y.; Suo, X.; Owen, G. S. (2007) Complexity Analysis for Information Visualization Design and Evaluation. p. 576-585. 